\title{
Patterns of medication use for the treatment of menopausal symptoms before and after the women's health initiative; implications for decision-making practices of women and women's health professionals
}

\author{
Chioma Uzoigwe Smith ${ }^{1}$, Srini Rajagopalan², Shiva Sajjan ${ }^{1}$, Shuvayu Sankar Sen ${ }^{1}$, Wenchen \\ Kenneth $\mathrm{Wu}^{3}$, Henry $\mathrm{Hu}^{1}$
}

${ }^{1}$ Global Health Outcomes, Merck \& Co., Inc., Whitehouse Station, USA; chiomaada@gmail.com
${ }^{2}$ Med Data Analytics, Inc., Milltown, USA;
${ }^{3}$ Department of Pharmacy Administration and Allied Health Sciences, St. John's University, Queens, USA.

Received 6 May 2011; revised 27 May 2011; accepted 10 June 2011.

\section{ABSTRACT}

Background: The Women's Health Initiative (WHI) published findings in 2002 that changed the perception of the use of hormone replacement therapy (HRT) for the reduction of cardiovascular risks. Menopausal women using HRT for the relief of vasomotor symptoms were advised to use the lowest possible dose of HRT over the shortest possible duration. Objective: This study sought to examine patterns of HRT use for the treatment of menopausal symptoms before and after the WHI among women at least 40 years of age. Methods: A retrospective analysis was performed on a total of 1367 women in the pre-WHI group and 6467 women in the post-WHI group using the U.S. General Electric (GE) Centricity electronic medical record database. Menopause diagnosis was assessed using ICD-9 codes. Clinical characteristics and medication use were assessed for women with at least 3 years of enrollment ( 1 year baseline, 2 years follow-up). Results: The proportion of women in the post-WHI group that initiated HRT was significantly less than that of women in the pre-WHI group $(31.3 \%$ vs. $56.9 \%$, respectively; $p$ $<0.001)$. Combination HRT use declined significantly $(21.9 \%$ pre-WHI cohort vs. $7.2 \%$ postWHI cohort, $p<0.001)$ among increases in nonHRT use, namely SSRIs (15.2\% pre-WHI cohort v. $22.3 \%$ post-WHI cohort, $p<0.001$ ) and tranquilizers (9.5\% pre-WHI cohort v. $15.8 \%$ post-WHI cohort, $p<0.001)$. Conclusion: The results of the WHI 2002 publication made an impression on the perception of HRT's role in the relief of menopausal symptoms. Decision-making on the part of women seeking treatment for vasomotor symptoms and women's health professionals demonstrates that despite HRT precautions, women continue to exhibit a need for HRT use. This study's findings suggest that women seeking treatment for menopausal symptom relief and women's health professionals continue to work together to find the appropriate balance between therapy use and adherence to therapy use guidelines.

Keywords: Women's Health Initiative (WHI); Hormone Replacement Therapy (HRT); Women's Health; Menopause

\section{INTRODUCTION}

Menopause is a normal part of aging signaling the end of menstruation and occurs 12 months after a woman's last menstrual period. Women typically menstruate until approximately 50 years of age. Signs and symptoms of menopause include irregular periods, decreased fertility, vaginal dryness, sleep disturbances, mood swings, increased abdominal fat, thinning hair, fatigue, headaches, depression, loss of breast fullness, urinary symptoms and hot flashes [1,2]. About $70 \%$ of women experience menopause symptoms of varying severity [2]. Those that experience severe symptoms may seek relief in the form of hormone replacement therapy (HRT), which has been widely prescribed to menopausal women for the past several decades. Several studies highlight the quality of life improvements HRT brings to women seeking relief from vasomotor symptoms [3-5].

While HRT relieves vasomotor symptoms such as hot 
flashes, it was also believed to provide cardioprotective effects for women at risk of coronary heart disease (CHD), a conclusion that was drawn from observational studies [6,7]. To better understand the effects of HRT, the Women's Health Initiative (WHI), was championed in 1991 to investigate the most common causes of death, disability and impaired quality of life in postmenopausal women [7]. The largest clinical trial to date, it included over 161,000 postmenopausal women 50 - 79 years of age. Patients were randomly assigned to placebo or one of 2 treatment arms: a combined oral HRT formulation-conjugated equine estrogen (CEE) $(0.625 \mathrm{mg})+$ progestin $(2.5 \mathrm{mg})$ - for women with an in-tact uterus, or CEE only for women who had undergone a hysterectomy.

The CEE + progestin arm was abruptly halted in 2002 after only 5.2 years of follow-up (the trial was originally to end after about 8.5 years of follow-up) because the risk of invasive breast cancer exceeded the projected boundary for the study [7]. Furthermore, results showed that among the 16,608 patients enrolled $(8,506$ patients in estrogen + progestin group, 8,102 patients in placebo group), absolute excess risks per 10,000 person-years attributable to estrogen + progestin therapy would result in 7 more cases of heart disease, 8 more cases of breast cancer, 8 more cases of stroke, 18 more cases of blood clots, and an increase in false positive mammograms [7]. Other results showed 6 fewer colorectal cancers and 5 fewer hip fractures. The WHI published these groundbreaking results in 2002 and the U.S. Food and Drug Administration (FDA) followed with its own recommendations for HRT use-HRT was not to be prescribed for CHD prevention. It was to be prescribed only for menopausal symptom relief at the lowest dose for the shortest duration possible.

The WHI publication and ensuing media coverage changed the perception of the HRT/CHD relationship in postmenopausal women [8]. Where it was once thought that HRT use served the dual purpose of relieving menopausal symptoms while providing cardiovascular protection, the new conclusions initiated cautious use of HRT. Consequently, prescribing practices changed, where high-dose combined HRT use realized a substantial decline, while low-dose HRT use increased [9,10]. Other studies reported a significant decline in HRT prescriptions and a significant decline in the average number of new HRT users per month less than one year after the WHI publication [6,11-14]. In addition, among HRT users, there was significant increase in discontinuation of HRT use post-WHI $[15,16]$. Women also turned to alternative therapies such as non-hormonal medications and other HRT formulations for which long-term health consequences were not well-known [17].

Several publications, however, have challenged the initial WHI findings, arguing that the WHI was premature to suggest the increased CHD risks of long-term HRT use $[18,19]$. These publications report that compared to women taking placebo, combined HRT shows increased CHD risk only during the first year of use, followed by much smaller risks thereafter (follow-up periods were for at least 6 years) [20,21]. Furthermore, HRT use did not significantly affect all-cause mortality rates and, paradoxically, reduced CHD risk was observed among younger women ( $\leq 60$ years of age) and women who initiated HRT no more than 10 years post menopause $[17,22,23]$. The risks of HRT use remain a contentious issue.

The emergence of research refuting the WHI results in 2002 begs the question of whether medical professionals and women using HRT prematurely gave up on the benefits HRT provides for the relief of vasomotor symptoms. Though use of high-dose HRT declined after the WHI publication in 2002, the data reflects that menopausal women were still using HRT in high proportions. Therefore, HRT remains a desired therapy of choice for menopausal women. This study revisits HRT utilization patterns before and after the WHI among patients newly diagnosed with menopause. It aims to add to the historic landscape of the changing trends in HRT use and support cautious - but not extreme-discretionary use of HRT for the relief of vasomotor symptoms.

\section{METHODS}

\subsection{Study Design}

This retrospective study was performed using the General Electric (GE) Centricity EMR system, a nationwide de-identified ambulatory electronic medical database of nearly 15 million patient records. Medication use patterns were assessed between 1999 and 2000 (preWHI) and 2003 and 2004 (post-WHI). Data collected for each cohort included one-year baseline and two years follow-up; therefore, the 1999-2000 cohort was followed through July 2002 (to account for a complete two years of follow-up) and the 2003-2004 cohort (post July 2002) was followed through 2007 (1998 data was collected but excluded for very low sample size). For diagnosis of menopause symptoms, ICD-9 codes of N95.1 and 627.2 were used. Generic product identifier (GPI) codes were used to evaluate the use of HRT, non-HRTs and other products ${ }^{1}$. ICD-9 codes were also used to identify the

${ }^{1}$ Other HRT included androgen + female hormones, topical sex hormones, monophasic preparations $(<50 \mathrm{mcg}$ estrogen and $\geq 50 \mathrm{mcg}$ estrogen), biphasic and triphasic preparations. Non-hormonal therapies included serotonin-norepinephrine reuptake inhibitors (SNRIs), selective serotonin reuptake inhibitors (SSRIs), non-barbiturate sedatives/hypnotics, tranquilizers, anti-epileptics, anti-hypertensives, antidepressants, and natural products. 
presence of comorbidities.

\subsection{Study Population, Eligibility and Dose Categories}

The study sample only included newly diagnosed menopausal women, who were free of menopausal diagnosis at the one-year baseline period and at least 40 years of age during the two follow-up periods (pre-WHI and post-WHI) between January 1, 1999 and December 31,2007 . A total of 1,397 women were in the pre-WHI group; 6,467 in the post-WHI group. HRT included only oral formulations and dose categories were defined as ultra low dose $(<0.3 \mathrm{mg}$ conjugated estrogen), low dose ( $0.3 \mathrm{mg}$ conjugated estrogen), medium dose $(0.45 \mathrm{mg}$ conjugated estrogen $)$, high dose $(0.625 \mathrm{mg}$ conjugated estrogen or $1 \mathrm{mg}$ or $1.5 \mathrm{mg}$ estrogen) and very high dose ( $1.25 \mathrm{mg}$ conjugated estrogen or $\geq 2 \mathrm{mg}$ estrogen).

\subsection{Statistical Analysis}

We used Chi-square tests to discern the statistical differences for categorical variables except for one instance in which Fisher's exact test was used because expected frequency was less than 5 in one cell count. Medication use was evaluated in the two-year follow up periods for each cohort (2000-2002 pre-WHI cohort, 2005-2007 post-WHI cohort) and defined as the first prescription filled on or after diagnosis date (1999-2007). Medications were categorized as HRT (estrogen and progestogen), other HRT (e.g., combined oral contraceptives of various regimens containing estrogen and progestogen, androgen + female hormones) and non-HRT (statins, anti-depressants, tranquilizers, etc). All analyses were performed using SAS version 9.1 and a $p$ value less than 0.05 was considered statistically significant.

\section{RESULTS}

Mean age at menopause diagnosis was 57.3 years \pm 10.9 in the pre-WHI group, 58.2 years \pm 11.0 in the post-WHI group (Table 1). There were significant decreases in the proportions of diabetes, dyslipidemia and hypertension after the end the WHI. The proportion of obese women also decreased significantly after the WHI. Approximately $80 \%$ of women in both cohorts were under 70 years of age.

Significantly fewer women in the post-WHI group initiated hormone therapy after menopause diagnosis than did those in the pre-WHI cohort $(31.3 \%$ vs. $56.9 \%$, respectively; $\mathrm{p}<0.001$ ) (Table 2 ). The use of combination therapy also precipitously declined among the post-WHI cohort. After the WHI's end, SNRIs, SSRIs and tranquilizers realized the most significant increases
Table 1. Descriptive characteristics of menopause patients in GE database 1998-2007 before (1999-2000) and after (2003-2004) the WHI.

\begin{tabular}{|c|c|c|c|}
\hline & $\begin{array}{c}\text { Pre-WHI } \\
\text { cohort } \\
\text { 1999-2000 }\end{array}$ & $\begin{array}{l}\text { Post-WHI } \\
\text { cohort } \\
2003-2004\end{array}$ & p-value \\
\hline Sample size (N) & $1397(17.8)$ & $6467(82.2)$ & \\
\hline $\begin{array}{l}\text { Age (yrs) } \\
\text { categories at index } \\
\text { date (mean, SD) }\end{array}$ & $57.34 \pm 10.85$ & $58.2 \pm 11.03$ & $<0.006$ \\
\hline $40-49(\mathrm{~N}, \%)$ & $402(28.8)$ & $1612(24.9)$ & \\
\hline $50-59$ & 473 (33.9) & $2304(35.6)$ & \\
\hline $60-69$ & 275 (19.7) & $1278(19.8)$ & \\
\hline $70-79$ & $190(13.6)$ & 924 (14.3) & \\
\hline $80+$ & $57(4.1)$ & $349(5.4)$ & \\
\hline $\begin{array}{l}\text { Baseline } \\
\text { comorbidities } \\
\text { Ankylosing spondylitis } \\
(\mathrm{N}, \%)\end{array}$ & $1(0.1)$ & $26(0.4)$ & 0.06 \\
\hline Depression & $105(7.5)$ & 489 (7.6) & 0.95 \\
\hline Diabetes & $151(10.8)$ & $357(5.5)$ & $<0.001$ \\
\hline Dyslipidemia & $218(15.6)$ & 814 (12.6) & 0.00 \\
\hline Heart failure & $4(0.3)$ & $16(0.3)$ & 0.79 \\
\hline Hypertension & $243(17.4)$ & $648(10.0)$ & $<0.001$ \\
\hline Obesity & $74(5.3)$ & $168(2.6)$ & $<0.001$ \\
\hline Osteoporosis & $52(3.7)$ & $190(2.9)$ & 0.12 \\
\hline Rheumatoid arthritis & $5(0.4)$ & $30(0.5)$ & 0.60 \\
\hline Spondylosis & $15(1.1)$ & $49(0.8)$ & 0.23 \\
\hline
\end{tabular}

Table 2. First medication after menopause diagnosis.

\begin{tabular}{lccc}
\hline & $\begin{array}{c}\text { Pre-WHI } \\
\text { cohort } \\
\text { 1999-2000 }\end{array}$ & $\begin{array}{c}\text { Post-WHI } \\
\text { cohort } \\
\mathbf{2 0 0 3 - 2 0 0 4}\end{array}$ & p-value \\
\hline $\begin{array}{l}\text { Sample size (N) } \\
\text { Hormone }\end{array}$ & 1154 & 6060 & \\
$\begin{array}{l}\text { Replacement Therapy } \\
\text { (HRT) (N, \%) }\end{array}$ & $624(56.9)$ & $1591(31.3)$ & $<0.001$ \\
Estrogen & $362(33.0)$ & $1148(22.6)$ & $<0.001$ \\
Estrogen + Progestogen & $240(21.9)$ & $363(7.2)$ & $<0.001$ \\
Progestogen only & $116(10.6)$ & $187(3.7)$ & $<0.001$ \\
Other HRT & $105(9.6)$ & $510(10.0)$ & 0.42 \\
Non-HRT & $79(7.2)$ & $374(7.4)$ & 0.37 \\
Antidepressants & $39(3.6)$ & $291(5.7)$ & 0.03 \\
Antiepileptics & $32(2.9)$ & $245(4.8)$ & 0.03 \\
Antihypertensives & $55(5.0)$ & $435(8.6)$ & 0.002 \\
Non-Barbiturates & $18(1.6)$ & $363(7.2)$ & $<0.001$ \\
SNRIs & $167(15.2)$ & $1133(22.3)$ & 0.003 \\
SSRIs & $104(9.5)$ & $800(15.8)$ & $<0.001$ \\
Tranquilizers & $2(0.2)$ & $32(0.5)$ & 0.11 \\
\hline $\begin{array}{l}\text { Other therapeutic } \\
\text { products }\end{array}$ & & & \\
\hline
\end{tabular}

aincludes all other gynecologic medications and other therapeutic products not containing estrogen or progesterone. 
in use $^{2}$. A significantly higher proportion of women in the post-WHI cohort initiated non-HRT post diagnosis than did women in the pre-WHI cohort $(66.1 \%$ vs. $42.0 \%$ respectively, $\mathrm{p}<0.001$ ) (data not shown).

As Table 3 shows, the proportions of women in the post-WHI cohort initiating lower doses of estrogen therapy after menopause diagnosis increased significantly compared to the proportions used by pre-WHI cohort women. Patients who changed HRT dose or switched to a different medication had shorter average duration of therapy (in days) on the initial HRT in the post-WHI cohort compared to the pre-WHI cohort and in both cohorts $46 \%$ of patients discontinued therapy (Table 4 and Table 5 ).

The incidence rates of menopause diagnosis between 1998 and 2007 are displayed in Figure 1. There appeared to be an increase in menopause diagnosis leading to the end of WHI trial. Most notable is the sizeable decrease in incidence from the trial's end (1.6 in 2002) to 2 years post-WHI (1.35). After 2004, the incidence rates remain relatively steady through 2007 .

Table 3. Estrogen dosing levels of first prescription after diagnosis.

\begin{tabular}{|c|c|c|c|}
\hline & $\begin{array}{c}\text { Pre-Cohort } \\
1999-2000\end{array}$ & $\begin{array}{c}\text { Post-Cohort } \\
\text { 2003-2004 }\end{array}$ & p-value \\
\hline Sample size (N) & 593 & 1497 & \\
\hline \multicolumn{4}{|l|}{$\begin{array}{l}\text { Estrogen } \\
\text { Potency }\end{array}$} \\
\hline $\begin{array}{l}\text { Ultra Low } \\
\text { Dose }\end{array}$ & $50(8.4)$ & $257(17.2)$ & $<0.001$ \\
\hline Low Dose & $46(7.8)$ & $293(19.6)$ & $<0.001$ \\
\hline Medium Dose & $0(0.0)$ & $109(7.3)$ & $<0.001$ \\
\hline High Dose & $357(60.2)$ & 714 (47.7) & $<0.001$ \\
\hline $\begin{array}{l}\text { Very High } \\
\text { Dose }\end{array}$ & $52(8.8)$ & $115(7.7)$ & 0.35 \\
\hline
\end{tabular}

Table 4. Pre-WHI cohort HRT average duration of therapy (in days) for all patients.

\begin{tabular}{cccccc}
\hline Switch & N & Mean & SD & Min & Max \\
\hline Persistent & 86 & 730 & 0 & 730 & 730 \\
Dose Change & 92 & 301.5 & 254.4 & 5 & 730 \\
Switch to other HRT & 12 & 286.8 & 287.2 & 29 & 730 \\
Switch to non-HRT & 145 & 300.6 & 278.6 & 5 & 730 \\
Discontinued & 289 & 178.2 & 173.1 & 1 & 685 \\
Switch & N & Mean & SD & Min & Max \\
\hline
\end{tabular}

${ }^{2}$ Though SNRIs and SSRIs are antidepressants, they are teased out of the antidepressants classification because they are commonly prescribed off-label to treat menopause symptoms.
Table 5. Post-WHI cohort HRT average duration of therapy (in days) for all patients.

\begin{tabular}{llllll}
\hline Switch & N & Mean & SD & Min & Max \\
\hline Persistent & 126 & 730 & 0 & 730 & 730 \\
Dose Change & 222 & 235.7 & 230.3 & 2 & 730 \\
Switch to other HRT & 38 & 224.7 & 244.3 & 2 & 730 \\
Switch to non-HRT & 469 & 213 & 224.5 & 2 & 730 \\
Discontinued & 736 & 176.7 & 174.4 & 1 & 697 \\
\hline
\end{tabular}

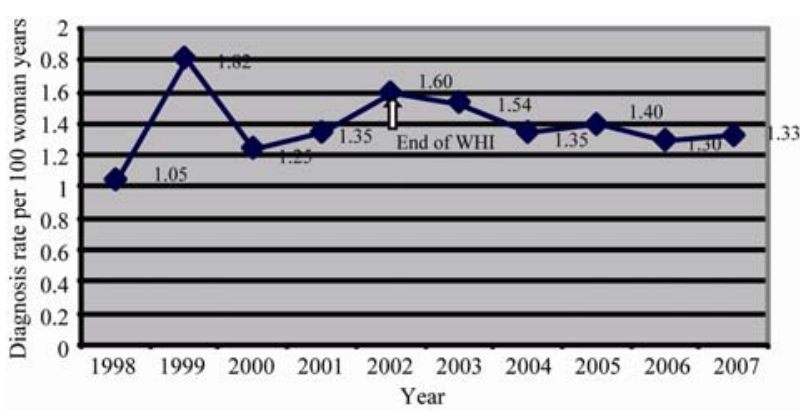

Figure 1. Menopause diagnosis rates (1998-2007).

\section{DISCUSSION}

In light of the publications that refute the initial findings of the WHI in 2002 [17-23], this publication highlights patterns of HRT use before and after the WHI in women newly diagnosed with menopause. By revisiting the issue of patterns of HRT use, we attempted to understand the impact the 2002 WHI publication had on the choices women made to treat their vasomotor symptoms.

Our data indicated significant decreased use of HRT overall between pre-and post-WHI cohorts, with the greatest decline in use among women using estrogen and progestin combined. High dosages of HRT fell out of favor post-WHI, leading to increases in use of lower dosages of HRT. Concordantly, use of non-HRTs, particularly SSRIs, SNRIs and tranquilizers, increased significantly after the WHI ended. These results are in alignment with previous studies that investigated HRT use trends before and after the end of the WHI [6,814,24,25]. A study documenting HRT use in postmenopausal women in the UK between April 2001 and September 2005 reported that the average proportion of women on HRT declined after the WHI interim results were published in July 2002 (28\% before July 2002 vs. $10.9 \%$ after July 2002) [13]. A 2008 Australian study reported a dramatic drop $(-55.4 \%)$ in the use of fixed combination (estrogen + progestogen) therapy in the 12 months following the end of the WHI estrogen + progestin arm [12]. A US-based study that sought to characterize the impact of the end of the WHI on HRT prescrip- 
tion patterns reported that after the WHI trial, women were less likely to initiate HRT [25]. Altogether, dissemination of the WHI findings may explain the precipitous decline in HRT use, particularly estrogen + progestin combinations $(22 \%$ of pre-WHI cohort, $7 \%$ of post-WHI cohort). A decline in fixed combination prescribing as reported by previous studies may have also contributed to this decline. In a Canadian study that used a healthcare database to assess the impact of the WHI publication on the rate of HRT prescriptions, results showed a significant increase post-WHI in the percentage of women using low-dose estrogen therapy $(<0.625 \mathrm{mg})$ where $8.3 \%$ of the pre-WHI cohort was using such therapy, compared to $14.8 \%$ of the post-WHI cohort ( $\mathrm{p}<$ $0.001)$ [6].

While the 2002 publication may have impacted diagnosis rates, resulting fear surrounding the safety of HRT and therefore, decreased doctor visits that would result in a diagnosis, may also have contributed to the incidence rate decline. Though depression was prevalent in about the same proportions in both cohorts, its presence was disproportionately low compared to the proportions of women using antidepressants (including SNRIs and SSRIs) for menopausal symptom relief. After the WHI trial's conclusion, many menopausal women sought alternative methods to relieve vasomotor symptoms [26]. After HRT, antidepressants are increasingly becoming next in line to treat symptoms of menopause. Some clinical trials have shown that antidepressants, most commonly those in the SSRI and SNRI classes, have reduced occurrences of hot flashes [26]. The benefits of off-label use of antidepressants to treat menopause symptoms are thought to be two-fold: 1) they treat the mood fluxes that accompany symptoms of menopause and 2) they relieve vasomotor symptoms [27]. Use of antidepressants to treat menopause symptoms appears to be controversial, especially for menopausal women who claim to experience no symptoms of depression or anxiety; however, this argument is beyond the scope of this analysis.

Our analysis shows significant declines in baseline comorbid conditions, including diabetes, dyslipidemia, hypertension and obesity, between the time periods in question. As we are not certain when women in our sample began using HRT, it can be speculated that the declines in CV risk-factors may be attributable to patient self-selection. In other words, the communication of HRT risks from the 2002 WHI publication may have discouraged women who had high CV risk factors from seeking treatment for menopausal symptoms; therefore, proportions of $\mathrm{CV}$ risk-factors in the post-WHI cohort may reflect underestimates. In the post-WHI cohort, average persistence mirrored that in the pre-WHI cohort (mean duration 730 days, each); however, dose changes and switches reflect shorter average duration of initial therapies among the post-WHI cohort. This suggests compliance recommended guidelines for HRT use, which may have encouraged higher satisfaction with use of lower-dose HRTs.

In each cohort, discontinuation of therapy was evident in a plurality of women $(46 \%)$, but this leaves a proportion of 54\% that did not discontinue HRT. Furthermore, discontinuation rates were the same for both time periods. This shows that despite the risks the WHI found, women still want to use HRT, but appear to be cautious about their choices. The changes seen may also reflect the publication's influence on women's health professionals who may have changed prescribing practices to help their patients seek relief of vasomotor symptoms.

The findings of this analysis should be interpreted in the context of study limitations. The GE Centricity database is an electronic health database in which physicians complete patient records; inherent limitations with this type of data collection include completeness of patients' records and human error. Missing information such as dosing, when a prescription was written, whether a prescription was filled or refilled, or the accuracy of comorbidity recording may be called into question. However, we believe missing information occurred in random fashion and unlikely resulted in systematic bias in findings. We assumed that the selected drugs taken after menopause diagnosis were actually for the relief of menopausal symptoms, though in cases where antidepressants are used, for example, we cannot discern if they were prescribed for menopausal symptoms or to treat symptoms of depression or other mental disorders. This analysis only considered oral HRT formulations and therefore, results cannot be generalized to populations of women using HRTs in other forms. Lastly, the two-year length of follow-up does not allow for generalization of results to women who have remained on HRT for longer durations.

Our results are consistent with studies that have demonstrated that menopausal women reacted quickly to ensure their health and well-being regarding HRT [6,8-14]. Given the trend that HRT use did not come to a complete halt, this study highlights that clearly there is still a need to find the proper balance between menopause symptom relief and exposure to health risks. To credit the WHI 2002 publication, women at high cardiovascular risk became cautious about their HRT use; however, the publication may have given women extreme pause towards the use of HRT, such that those who still sought relief of vasomotor symptoms unnecessarily deprived themselves of the level of relief HRT could provide. 


\section{CONCLUSIONS}

The results of the WHI stirred faith in the once-assumed cardioprotective effects of HRT for menopausal women. Since the WHI ended, use of high-dose HRT declined, while use of non-HRT, such as antidepressants, increased. The results of this study highlight the impact the WHI had on the choices physicians and women made to treat vasomotor symptoms, as well as the importance women place on their health outcomes during the stage of amenorrhea. This study's findings suggest that women seeking treatment for menopausal symptom relief and women's health professionals continue to work together to find the appropriate balance between therapy use and adherence to therapy use guidelines.

\section{REFERENCES}

[1] Mayo Clinic (2010) Menopause symptoms. http://www.mayoclinic.com/health/menopause/DS00119/ DSECTION $=$ symptoms

[2] Nihira, M.A. (2010) Menopause guide. http://www.webmd.com/menopause/guide/understanding -menopause-basics

[3] Birkhauser, M.H. (2009) Quality of life and sexuality issues in aging women. Climacteric, 12, 52-57. doi:10.1080/13697130903013163

[4] Van Dole, K.B., Williams, R.E., Brown, R.S., Gaynes, B., Devellis, R. and Funk, M.J. (2010) Longitudinal association of vasomotor symptoms and psychosocial outcomes among postmenopausal women in the United States: A population-based study. Menopause, 17, 917-923. doi:10.1097/gme.0b013e3181d824c8

[5] Welton, A.J., et al. (2008) Health related quality of life after combined hormone replacement therapy: Randomised controlled trial. British Medical Journal, 337, a1190. doi:10.1136/bmj.a1190

[6] Guay, M.P., et al. (2007) Changes in pattern of use, clinical characteristics and persistence rate of hormone replacement therapy among postmenopausal women after the WHI publication. Pharmacoepidemiology and Drug Safety, 16, 17-27. doi:10.1002/pds.1273

[7] Rossouw, J.E., et al. (2002) Risks and benefits of estrogen plus progestin in healthy postmenopausal women: principal results from the women's health initiative randomized controlled trial. Journal of the American Medical Association, 288, 321-333.

[8] Usher, C., et al. (2006) Effect of clinical trial publicity on HRT prescribing in Ireland. European Journal of Clinical Pharmacology, 62, 307-310. doi:10.1007/s00228-005-0083-x

[9] Hing, E. and Brett, K.M. (2006) Changes in U.S. prescribing patterns of menopausal hormone therapy, 2001-2003. Obstetrics \& Gynecology, 108, 33-40. doi:10.1097/01.AOG.0000220502.77153.5a

[10] Wei, F., et al. (2005) Changes in women's use of hormones after the women's health initiative estrogen and progestin trial by race, education, and income. Journal of the National Cancer Institute Monographs, 35, 106-112. doi:10.1093/jncimonographs/lgi047

[11] Faber, A., et al. (2005) Dramatic change in prescribing of hormone replacement therapy in The Netherlands after publication of the million women study: A follow-up study. British Journal of Clinical Pharmacology, 60, 641-647. doi:10.1111/j.1365-2125.2005.02502.x

[12] Main, P. and Robinson, M. (2008) Changes in utilisation of hormone replacement therapy in Australia following publication of the findings of the women's health initiative. Pharmacoepidemiology and Drug Safety, 17, 861868. doi: $10.1002 / \mathrm{pds} .1605$

[13] Menon, U., et al. (2007) Decline in use of hormone therapy among postmenopausal women in the United Kingdom. Menopause, 14, 462-467. doi:10.1097/01.gme.0000243569.70946.9d

[14] Newton, K.M., et al. (2005) The impact of comorbidities on hormone use. After the 2000 release of the Women's Health Initiative. Journal of General Internal Medicine, 20, 350-356. doi:10.1111/j.1525-1497.2005.04059.x

[15] Bestul, M.B., et al. (2004) Impact of the women's health initiative trial results on hormone replacement therapy. Pharmacotherapy, 24, 495-499. doi:10.1592/phco.24.5.495.33349

[16] Gerend, M.A., et al. (2006) Women's use of hormone therapy before and after the women's health initiative: A psychosocial model of stability and change. Preventive Medicine, 43, 158-164. doi:10.1016/j.ypmed.2006.04.008

[17] Rossouw, J.E., et al. (2007). Postmenopausal hormone therapy and risk of cardiovascular disease by age and years since menopause. Journal of the American Medical Association, 297, 1465-1477. doi:10.1001/jama.297.13.1465

[18] Stevenson, J.C. (2009) Hormone replacement therapy and cardioscular disease revisited. Menopause International, 15, 55-57. doi:10.1258/mi.2009.009018

[19] Anderson, G.L., et al. (2004) Effects of conjugated equine estrogen in postmenopausal women with hysterectomy: The women's health initiative randomized controlled trial. Journal of the American Medical Association, 291, 1701-1712. doi:10.1001/jama.291.14.1701

[20] Manson, J.E., et al. (2003) Estrogen plus progestin and the risk of coronary heart disease. The New England Journal of Medicine, 349, 523-534. doi:10.1056/NEJMoa030808

[21] Du, Y., et al. (2007) Differences in menopausal hormone therapy use among women in Germany between 1998 and 2003. BMC Womens Health, 7, 19. doi:10.1186/1472-6874-7-19

[22] Manson, J.E., et al. (2007) Estrogen therapy and coronary-artery calcification. The New England Journal of Medicine, 356, 2591-2602. doi:10.1056/NEJMoa071513

[23] Hsia, J., et al. (2006) Conjugated equine estrogens and coronary heart disease: The women's health initiative. Archives of Internal Medicine, 166, 357-365. doi:10.1001/archinte.166.3.357

[24] Parazzini, F. (2008) Trends of determinants of hormone therapy use in Italian women attending menopause clinics, 1997-2003. Menopause, 15, 164-170.

[25] Wegienka, G., et al. (2006) Menopausal hormone therapy in a health maintenance organization before and after women's health initiative hormone trials termination. 
Journal of Women's Health, 15, 369-378.

doi:10.1089/jwh.2006.15.369

[26] Barton, D.L., et al. (2003) Pilot evaluation of citalopram for the relief of hot flashes. The Journal of Supportive Oncology, 1, 47-51.
[27] Mills, D. (2010) Antidepressants for menopause symptoms: Pros and cons.

http://www.womentowomen.com/menopause/antidepress antsinmenopause.aspx\#offlabel 